\title{
Improving Web Movie Recommender System Based on Emotions
}

\author{
Karzan Wakil \\ Computer Department \\ Institute of Training and Educational Development-Sulaimani \\ Sulaimaniyah-Iraq \\ Karwan Ali \\ Computer Science Department \\ University of Human Develpment \\ Sulaimaniyah-Iraq
}

\begin{abstract}
Recommender Systems (RSs) are garnering a significant importance with the advent of e-commerce and ebusiness on the web. This paper focused on the Movie Recommender System (MRS) based on human emotions. The problem is the MRS need to capture exactly the customer's profile and features of movies, therefore movie is a complex domain and emotions is a human interaction domain, so difficult to combining together in the new Recommender System (RS). In this paper, we prepare a new hybrid approach for improving MRS, it consists of Content Based Filtering (CBF), Collaborative Filtering (CF), emotions detection algorithm and our algorithm, that presented by matrix. The result of our system provides much better recommendations to users because it enables the users to understand the relation between their emotional states and the recommended movies.
\end{abstract}

Keywords-movie recommender system; collaborative filtering; content based filtering; emotion; $\mathrm{CF} ; \mathrm{CBF} ; \mathrm{MRS}$

\section{INTRODUCTION}

RSs can be described as the software tools and techniques offering recommendations for items to be of use to a user [1]. Users today in the world with the internet and its associated information explosion are facing with the problematic situations that have too many options. Right from looking for a restaurant to looking for a good investment selection, there is huge information available. As assisting to the users as they can deal with this information burst, companies have organized $\mathrm{RSs}$ to direct them. The research in the area of RSs has been going on for several decades now, but the interest remaining elevation due to the plenty of practical applications and the problem rich domain. There are many active examples of online RSs that are implemented such as a RS for books at (Amazon.com), Library for movies at (MovieLens.org), CDs at (CDNow.com), and so on [2].

Recommender systems are now common both commercially and in the research community, where many methods and techniques have been suggested for providing recommendations. In many cases, a system designer that wishes to employ a RS must choose between a set of candidate

\author{
Rebwar Bakhtyar \\ Information Technology Department \\ Computer Science Institute-Sulaimani Polytechnic University \\ Sulaimaniyah-Iraq
}

Kozhin Alaadin

Computer Science Department

University of Human Develpment

Sulaimaniyah-Iraq

approaches. A first step towards selecting the appropriate algorithm is to decide which properties of the application to focus upon when making this choice. Indeed, RSs have a variety of properties and attributes that may affect user experience, such as accuracy, robustness, scalability, and so forth [3]. Movie recommendation system which is recommends movies to users based on their personal information and their answers to questions based on movies [2].

The proposed system for the movie recommendation uses both $\mathrm{CF}$ and $\mathrm{CBF}$. In order to provide reliable recommendation, the RSs need to capture the customer needs and preferences exactly into the user profile. However, for subjective and complex products such as movies, music, news, user emotion plays an unexpected critical roles in the decision process. As the traditional model of user profile does not take into account the influence of the user emotion; the RS cannot recognize and capture the repetitively changing preferences of the user's like. Emotional status determination of the user is the main role of this algorithm, as it determines the state of the user's emotion according to three chosen colors by the user. In order to do so, it will analyses the color sequence by using the follow logic; if at least two of three chosen colors indicate the same emotion and this emotion becomes the current emotional state of the user [4-5].

The future is a viable opportunity to introduce a contextualized personalized and emotional RS with the ability to implement Multi-Agent System (MAS), sports among other domains. It makes such retrieval system necessary that along with the task of information gathering, could also involve in selective filtering as per the interests and emotions triggered by the information in the subject. For instance, today's hectic routine creates impediment for people in remaining up-to-date with respect to ones' social circle or world happenings [6]. It necessitates the embedding of users' intentions, their social networking habits and community trends into RS application. For accomplishing this purpose MAS intended to relay selective information to the subject is ought to be used [7].

The problem is the movie RSs need to capture exactly the customer's profile and movie features because the movie is a 
complex domain, and emotion is a human interaction domain, so difficult to combining in the new recommender system. In this paper, we will apply matrix for integrating movie recommendation by hybrid approach, which consists of $\mathrm{CBF}$ and $\mathrm{CF}$ system with emotions detection algorithm and our algorithm. This system much better recommendations to users because it enables the users to understand the relation between their emotional states and the recommended movies.

This paper organized as follows: section 2 explains the background work of the movie RS and RSs based on emotion. Section 3 explained the recommendation approaches, human emotions, and combination the approaches. Section 4 demonstrates our algorithm to improving MRS based on emotion. In the section 5 presents some concluding remarks and points to future works.

\section{BACKGROUND AND RELATED WORK}

In the last years, many RSs designed, and several RSs based on human emotions completed. This section presents some works that published by researchers.

According to [8], the CBF systems done each item by its related features. They learn a profile of the user's interests based on the features present in the items that the user has rated. The systems make recommendations by considering the description of the items that has been rated by the user and the depiction of items to be recommended. The recommendations can be made even if the system has received a small number of ratings, as the recommendations are based on product features. However, CBF systems are inadequate by the features that are explicitly related with the objects that they recommend [9].

By using MAS approach and taking advantage from some techniques of some other fields like Artificial Intelligence (AI) and Affective Computing (AC) this study Costa, H. and L. Macedo,2013 have been answered to the growing demand on RS. More precisely, it cope the privation of emotion-based RS, especially in relation to real-time textual information. While there is no study that related to this kind of the RS for Portuguese or even for English. This work will be focused in the development of a RS capable of filtering irrelevant and emotionless news to the user, by using a MAS approach [10].

Quijano-Sanchezat.al, 2011 have been issued a movie recommender system as an application in name Happy Movie (HM), for group of people which it is integrated with the social network face book (FB). They tried through HM to diminish a certain limitations in existing group RSs, like obtaining the user's profile or offering trading methods for users in order to reach a final agreement. The utilized method to make the group recommendation is based on three important features: personality, social trust and memory of past recommendations. Eventually, they simulate in a more realistic way the argumentation process followed by groups of people when deciding a joint activity [11].

Yang, and Yuan, 2010 presented a novel recommender service system to capture tourists' expectations (emphasizing on emotional needs) and to meet their satisfactions by recommending desirable attractions and SMEs in destination regions. Image, as a fundamental element and an operant resource, has been cultivated to be the uniform representation for tourists' expectations, destinations, and SMEs. In the system, the module constructs the images in three formats through the analysis of data; the interaction module and adaption module monitor the expected changes to images through the interactions between roles, and the unexpected changes caused by occasional environmental/social events respectively [12].

Menezes, and Tagmouti, introduced an Emotion-based Movie Recommender System (E-MRS) as a solution to this problem. The objective of E-MRS is to provide adapted and personalized suggestions to users using a combination of $\mathrm{CF}$ and content-based techniques. The recommendation is based on inferences about a user's emotions and preferences, as well as opinions of other similar users. In their paper discuss the system design and implementation, as well as its evaluation procedure. We believe that our system provides much better recommendation to users because it enables the users to understand the relation between their emotional states and the recommended movies [5].

Rajenderan, A, 2014 complete a project is to create a movie RS that uses human emotion as the basis of recommendation. The system will observe a user while they watch a portion of content, and then analyze the data that is their facial expressions and heart rate over time. With a sufficient database, their emotion data can be compared with those of other users, and recommendations can be provided based on reactions to the content [13], furthermore explains the landscape of actual and possible hybrid recommenders, and introduces a novel hybrid, a system that combines content boosted recommendation and $\mathrm{CF}$ to recommend restaurants [14].

Hong and $\mathrm{Zhu}$, in their study proposed a novel method to measure users' preference on movie genres, and utilize Pearson Correlation Coefficient (PCC) to calculate the user similarity. For genre preference, regularization they used matrix factorization framework. Experimental results on Movie Lens data set demonstrate that the approach performs well. Their method can also be used to increase the genre variety of recommendations to some extent [15], moreover many articles for improving movie recommender system and emotion done, see $[10,16-20]$.

However many RSs for solving complexity of MRS was prepared, also they used hybrid approach. For developing MRS based on human emotions need to enhanced through new techniques or merging two or more techniques, in the next sections we explain and prepare a new hybrid approach to improving MRS.

\section{RECOMMENDATION APPROACHES AND EMOTIONS}

There are three major methods or approaches for RS: CF, $\mathrm{CBF}$ and Hybrid approach which combines the previous two methods. The first experiments in the RS area adopted pure CF approaches. Many researcher used CBF techniques, which described and utilized are borrowed from the field of information retrieval. However, CBF differs from information retrieval in the manner in which the interests of the users are represented. $\mathrm{CBF}$ tries to model the user's long-term interests instead of using a query of an information filtering system. 
There are other systems that utilize content-based filtering to help users find information on the web. This section explains the RSs and emotions that used with MRS.

\section{A. Recommendation System (RS)}

RSs are information search tools that have been recently proposed to cope with the "information overload" problem, the typical state of the web user, of having too much information to make a decision or remain informed about a topic. In fact, users who are approaching a commerce website (e.g., Amazon), or a content website (e.g., cnet.com or visitfinland.com) for collecting information about a product or service, or simply a topic (e.g., Lapland) could be overwhelmed by the quantity of the relevant pages and ultimately the information displayed in these websites. In order to address this problem RS have been proposed.

The core computational task of an RS is to predict the subjective evaluation a user will give to an item. This prediction is computed using a number of predictive models that have a common characteristic, they exploit the evaluations/ratings provided by users for previously viewed or purchased items. Based on the particular prediction technique being employed, recommender systems have been classified into the following four main categories [9], Fig.1 shows a recommend system work.

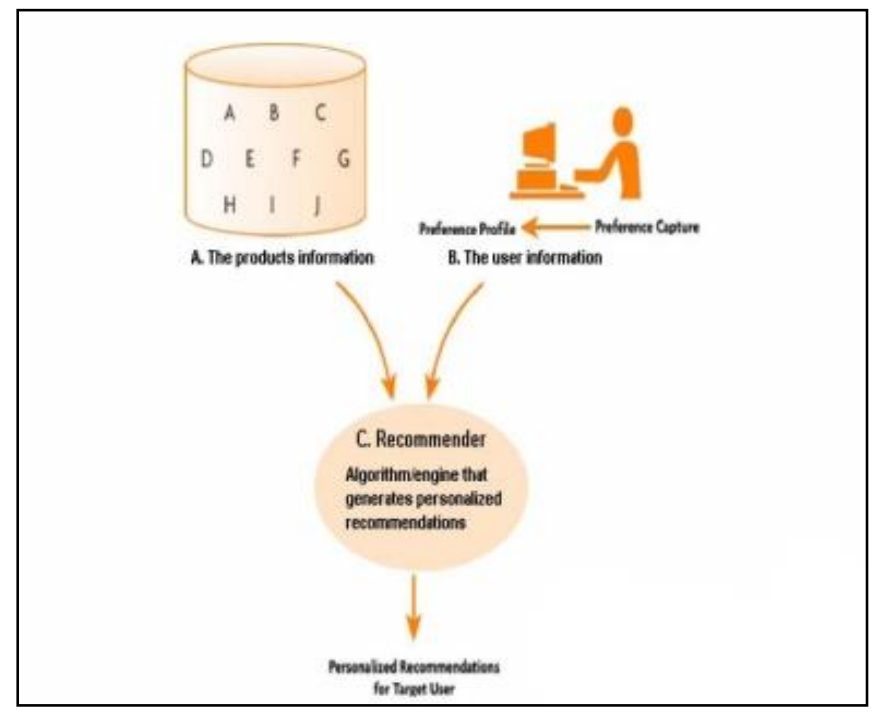

Fig. 1. Recommender System [21]

\section{B. Objective from Recommender System}

Prime objective of RS is to deliver to the user most relevant content as determined by ratings and previous consumption, in order to maximize positive user experience. Information overload is one of the main motivational factors behind this project the application of which could result in relevant content, thereby cementing the quality of recommendations. As opposed to 100-200 probably relevant recommendations, through the system under consideration we focus on delivering limited recommendations; though of higher quality reflects in our guarantee of the recommendations being more relevant [22].

\section{Recommendation Approaches}

There are many approaches that are used in recommender systems; Fig. 2 shows the popular techniques of the RS.

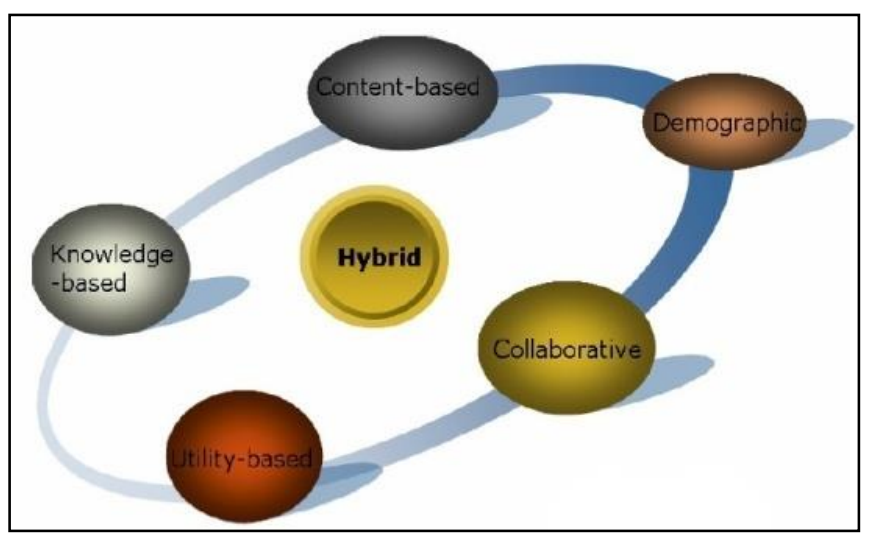

Fig. 2. Recommendation Techniques [9]

\section{1) Content-Based Filtering (Item-Item Comparison)}

CBF compares items against either user's interest profile or query derived from content. Strength of the technique over CF lies in recommendation delivery even when ratings received are lesser or none at all, as long as there is certain information pertaining to each item in the system. Each item however must be characterized in alignment with the features in user's profile. These descriptive features are either acquired or engineered. One paper introduced p-tango method, which uses both; content-based and collaborative filtering, by linear combination to create more effective filter than that produced through either method alone [4].

\section{2) Collaborative Filtering (User-User Comparison)}

It is also known as user-user contrast. One of the widely used recommendation methods is CF. Its basic idea is identification of likeminded users along with their crossrecommended items; items that likeminded users have liked, but the user on the receiving end has not consumed. Increasing popularity of this method could be attributed to the music industry where it is capitalized by such well-known websites as Pandora (www.pandora.com). One of the advantages it has is that it refrains from heavy calculations and so can produce highly accurate recommendations for a significant number of users in a timely manner. As the method draws on user-user similarity information to determine and pair likeminded users; users who are likely to agree rating of rate some specific content, it does not require any type of description regarding content as it only uses users' ratings for evaluation of their degree of agreement. User-user similarity information includes rating given by both users. Rating differences that determine user like-mindedness are stored in a database to intercept similar users when generating recommendations.

In [23] suggests a good structure for the similarity values in a table called a user-user matrix. The recommendations can now be produced by few variations of methods. For example find the most often rated or highest rated items on average from the users $\mathrm{N}$ closest neighbors that he has not seen or rated himself. 
What we however decided to do was a method inspired by the Group Lens team from [8]. By using the top $N$ nearest neighbor's ratings and similarity values, we can estimate a predicted rating by using a weighted average of the neighbor ratings. The highest predicted ratings will then give us a good idea of the user's interest and have shown to produce high quality recommendations. In-spite of the method's growing popularity there are two main problems that are generally associated with collaborative methods. The first issue is caused by new users in the systems which have not submitted any ratings. The system is therefore unable to find any qualified user-neighbors' and thereby also unable to predict any ratings based on that. This problem is called Cold-start problem. A simple solution to the problem is not to offer recommendations to users that have not submitted any ratings or perhaps fewer than some minimum number of ratings. The other perhaps more relevant problem is called the first-ratter problem. This problem is caused by new items in the systems that understandably have not received any ratings.

\section{3) Combination Recommendation Approaches}

Utilizing different methods are often related to the different recommendation issues. These problems are caused by four main instances that we try to avoid them by combining collaborative and content-based techniques to compensate each other downsides. The main causes are new items, new users that understandably have not submitted or received any ratings. Sparsely due to large data sets and little overlapping between users rating and finally averaging effect e.g. due to long time content consumption and poor system design [16], as shown in Fig 3.

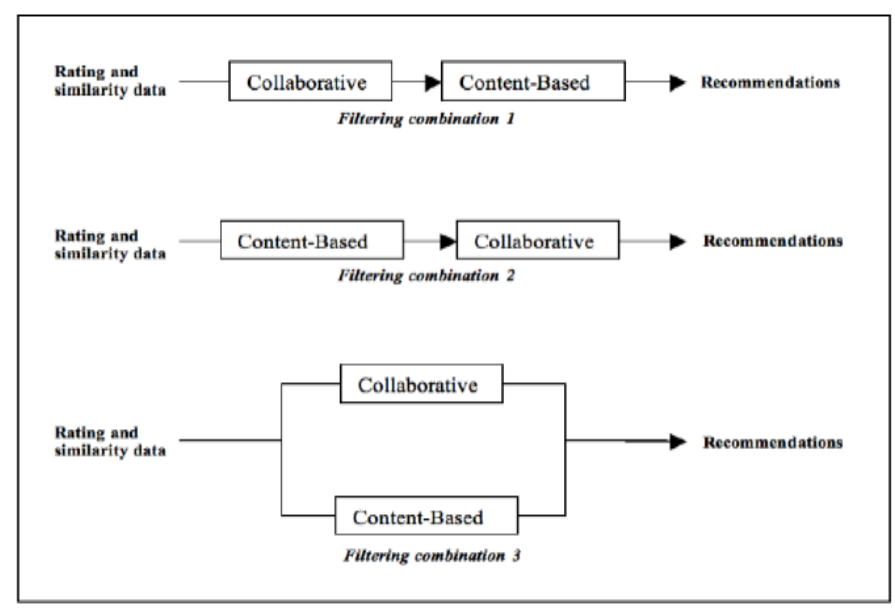

Fig. 3. Different Ways of Combining CF and CBF [16]

\section{Emotion States}

Various aspects of emotion are measurable including selfreported individuals' feelings, autonomic system reactions, neurological changes, and bodily actions inclusive of facial movements. Others nevertheless continue to argue presence of universal fundamental emotions; emotions that could be universally recognized, and their existence due to evolutionary pressure. For instance, cognitive processes and autonomic changes during fear induce flight response in human preparing them to run away from the threat or danger. A scientific evidence is highlighting five different emotions at least (anger, fear, disgust, sadness, happiness) being noticeably different considering the activation of different combinations in the brain supports the above phenomenon [24].

Emotion is a state usually triggered by any significant event characterizing importance of some level to the subject or user. It normally includes (a) conscious mental state having discernable quality of feeling directed towards any object, (b) some kind of bodily perturbation, (c) identifiable expressions on the face, tonality of the voice, and gesture (d) readiness to indulge in certain types of actions[25]. Emotion can be identified as a series of changes in the state, the changes that are way inter-dependent and synchronized in a way in response to evaluations to which relevance of internal or external stimulus are subjected.

People or viewers watch and experience movies everyday with affective response. They depict joy when watching a comedy and sadness while watching romantic movie late into the night. Another way to prove this is when somebody is in love; they would like to watch romantic movies that generally follow happy endings. However, when that same person is sad, they might watch fast-paced action flick as it could improve their feelings through subversion of their sadness that is triggered by their active involvement in the fast-paced movie to understand what is happening. Fast-paced movies usually overcome feelings of viewers; usually negative, as they indulge in decoding the scenarios unraveling in front of them in speed.

Another approach that could be utilized is learning the rules with the help of training. In this scenario, the user could be asked and required to answer several questions aided by the use of questionnaire (movie-emotion). The answer or user response to the questionnaire would indicate the preferences; with respect to movie, of the subject as per his emotions. It could be intercepted through such questions as the kind of movies one would like to watch when in the state of sadness, happiness etcetera. This method is usually chosen to be used for the creation and development of basic rules pertaining to the system focused in this assignment. Quality and quantity of the developed rules is imperative to be improved over a period with increasing number of users enrolling up for the system.

\section{METHODOLOGY AND SYSTEM FRAMEWORK}

This section explained the progress of a new movie recommender system based human emotions, which consist of $\mathrm{CF}$ and $\mathrm{CBF}$ after mixing emotion detection algorithm with our method to improvement the system.

The system framework consists of five phases as shown in Fig.4, in the first phase the users should be make a registration and chose three colors to selecting emotion state. In the second phase the system calculate initial rating and allow users to rating the movies from 1 to 5 , in the third phase system calculate the similarity between users and items, in the fourth phase the system predicting the user's rating through hybrid $\mathrm{RS}$, in the last phase the system sort the list of movies that recommended for the target user. 


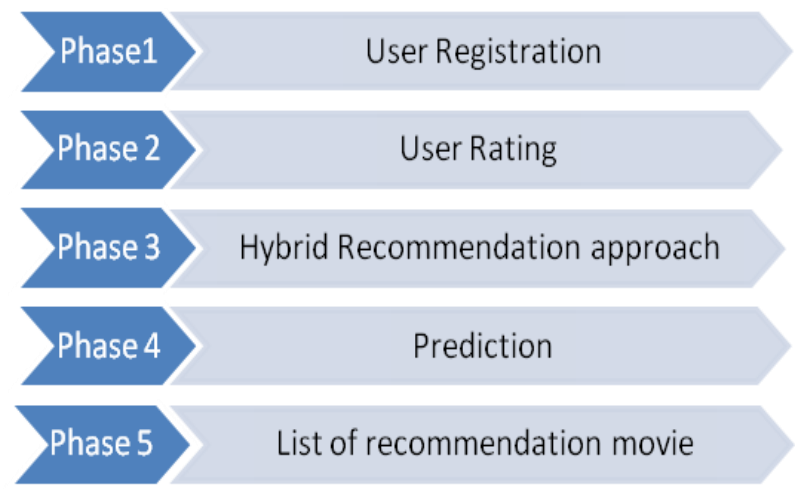

Fig. 4. System Framework

Phase 1: The users must start working in a special account in the system and enter the required information upon it such (id, user name, password, email, choosing three colors), then the three colors converted to emotion state by the emotion detection algorithm as shown in Fig.5.

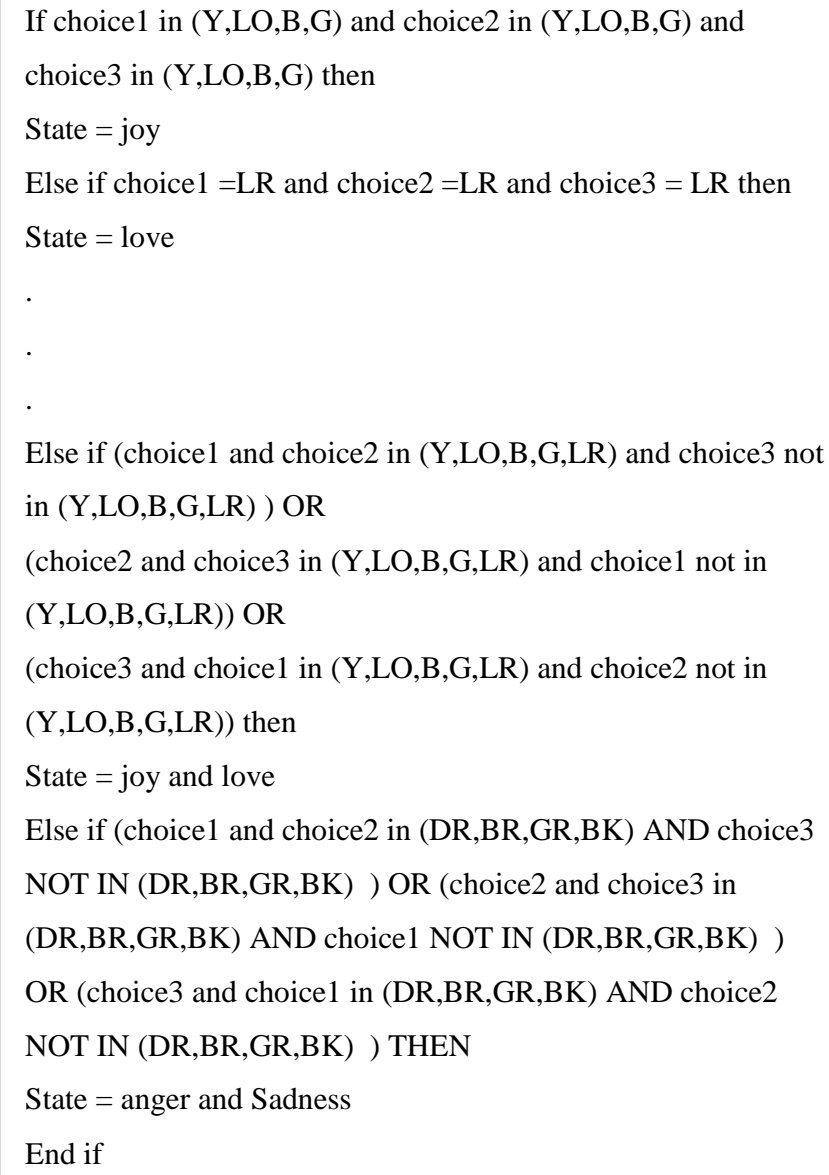

Fig. 5. Emotion Detection Algorithm

Phase 2: After registration the users can evaluate the movies by rating movies from 1 to 5 . The users after seen the part or the whole movie will decide the movie according to his/her like, then rated between 1 to 5 , as shown in Table 1 .
TABLE I. USER-ITEM MATRIX RATING

\begin{tabular}{|c|c|c|c|c|c|c|c|}
\hline & $\stackrel{\vec{\Xi}}{\stackrel{\Xi}{ \pm}}$ & $\stackrel{N}{\Xi}$ & $\stackrel{m}{\Xi} \underset{\Xi}{\Xi}$ & $\stackrel{+}{\Xi}$ & $\stackrel{\text { Ln }}{\Xi}$ & 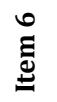 & 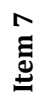 \\
\hline User 1 & 4 & 3 & 0 & 2.5 & 3.5 & 5 & 0 \\
\hline User 2 & 5 & 4 & 0 & 4.5 & 2.5 & 0 & 0 \\
\hline User 3 & 0 & 2.5 & 3 & 0 & 1 & 0 & 1.5 \\
\hline User 4 & 0 & 0 & 0 & 2.5 & 0 & 3.5 & 4 \\
\hline User 5 & 1 & 0 & 5 & 0 & 0 & 0 & 0 \\
\hline
\end{tabular}

Phase 3: This phase calculates a new hybrid recommender system that consists of $\mathrm{CF}$ and $\mathrm{CBF}$ with human emotions and our algorithm as shown in Fig.6.

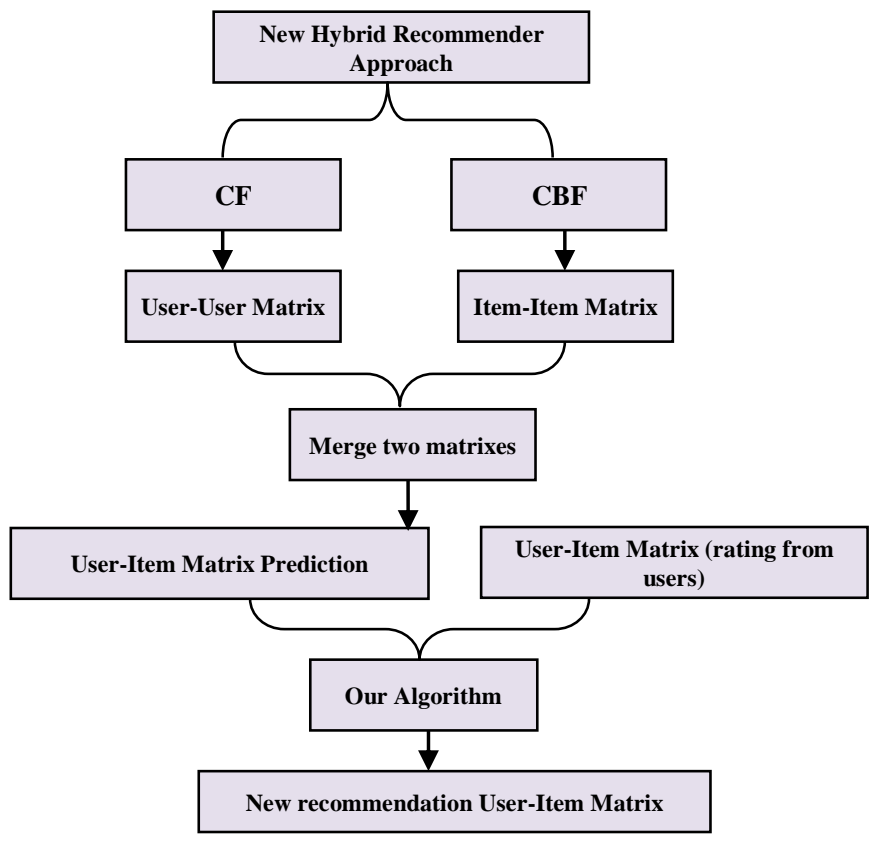

Fig. 6. New Hybrid Recommender System

In the following explained and calculated the steps of a new hybrid recommender system:

\section{A. Calculation User-User Matrix (CF)}

$\mathrm{CF}$ is a recommendation method used to finding similarity between users, as explained in section 3 , we can find similarity between users through equation (1).

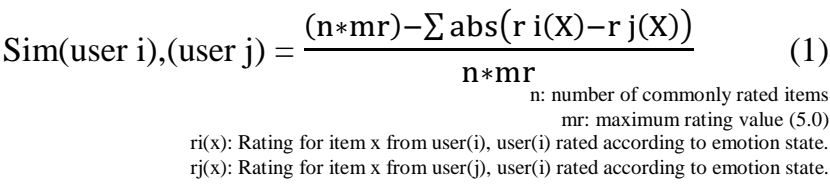

Example 1: Let's assume that two users in the system have submitted a few ratings, and from those ratings we want to compare how 'likeminded' these two users are. From all the ratings they have submitted only the following 5 items are overlapping, meaning items that both have rated, as shown in Table 2. 
TABLE II. USER RATING

\begin{tabular}{|c|c|c|c|}
\hline & User 1 & User 2 & Offset \\
\hline Item 1 & 4.5 & 2 & 2.5 \\
\hline Item 2 & 3 & 2 & 1 \\
\hline Item 3 & 2.5 & 5 & 2.5 \\
\hline Item 4 & 2 & 5 & 3 \\
\hline Item 5 & 1.5 & 4.5 & 3 \\
\hline Total & & & 12 \\
\hline
\end{tabular}

After applied equation (1), the similarity between user 1 and user 2 is $52 \%$ as calculated the following:

$$
\operatorname{Sim}(\text { user } 1),(\text { user } 2)=\frac{5 * 5-12}{5 * 5}=\frac{25-12}{25}=0.52=52 \%
$$

The similarity between users to get to the best result, and so when the user does a search for movies who likes to watch. To get the best result for users, we arrange movies by selected colors and the similarity between users.

Example 2: Let's assume that five users make ratings for five items, after finding the similarity between users by using equation (1), for easy present we use matrix for showing similarity between users as shown in Table 3 .

TABLE III. USER-USER SIMILARITY MATRIX

\begin{tabular}{|c|c|c|c|c|c|}
\hline & User1 & User2 & User3 & User4 & User5 \\
\hline User1 & 1 & 0.52 & 0.36 & 0.44 & 0.5 \\
\hline User2 & & 1 & 0.58 & 0.67 & 0.32 \\
\hline User3 & & & 1 & 0.65 & 0.83 \\
\hline User4 & & & & 1 & 0.78 \\
\hline User5 & & & & & 1 \\
\hline
\end{tabular}

Moreover Fig 7 shows the php code for finding similarity user-user.

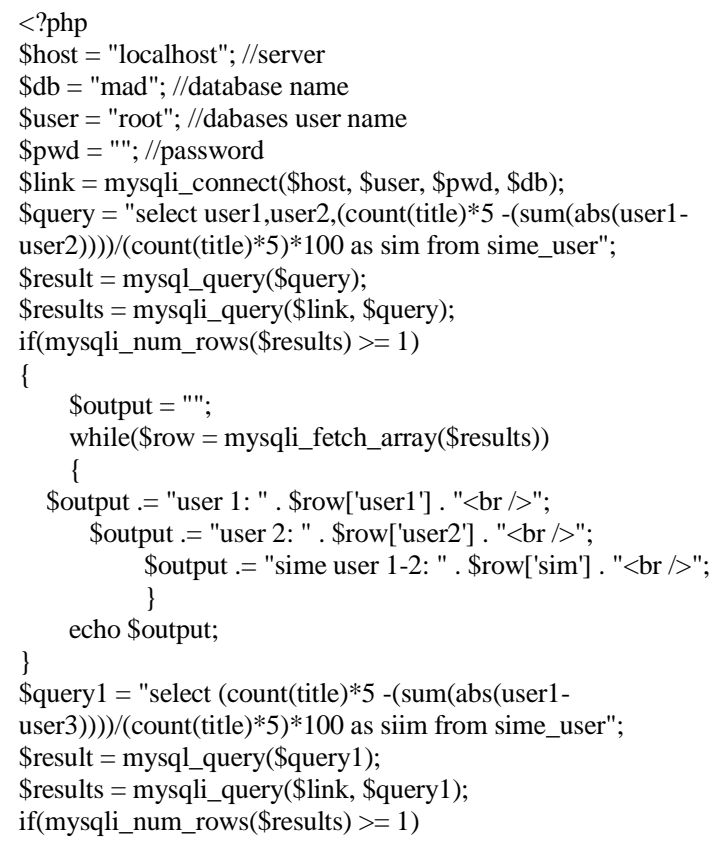

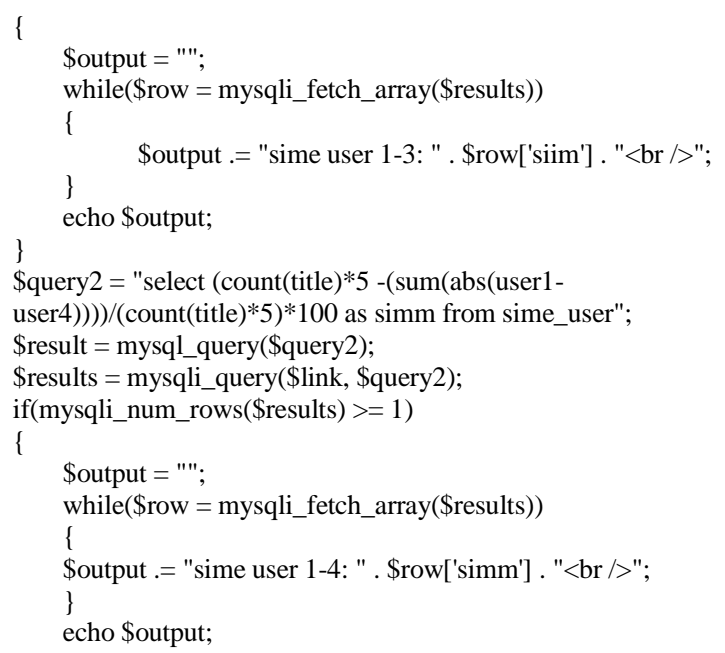

Fig. 7. php code for similarity user-user

\section{B. Calculation Item-Item Matrix (CBF)}

$\mathrm{CBF}$ is a recommendation method used to finding similarity between items, as explained in section 3, Calculating similarity between item-item should be defined item's profile; this attributes for a movie selected (title, genre, colors, country, and cast) as shown in Table 4.

TABLE IV. AN EXAMPLE OF AN ITEM-PROFILE FEATURING: GALDIATOR (2000)

\begin{tabular}{|l|l|}
\hline Title & Gladiator (2000) \\
\hline Genre & Action, Adventure, Drama \\
\hline Colors & Black, red \\
\hline Country & USA \\
\hline Cast & $\begin{array}{l}\text { Russell Crowe, Joaquin Phoenix, Connie Nielsen, Oliver } \\
\text { Reed, Richard Harris, } \\
\text { Derek Jacobi, Djimon Hounsou, David Schofield, John } \\
\text { Shrapnel, Tomas Arana, } \\
\text { Ralf Moeller, Spencer Treat Clark, David Hemmings, } \\
\text { Tommy Flanagan,... and so on. }\end{array}$ \\
\hline
\end{tabular}

The attributes listed in Table 4 is a typical item-profile which can used to compare how much alike items are. But the main concern in this field should be how selected the right attributes and which attributes describe the content in the best possible way. Assuming that we have two items with a number of attributes, each similarity between them is calculated using the following equation (2).

$$
\begin{array}{r}
\operatorname{Sim}(\operatorname{item}(\mathrm{i}), \operatorname{item}(\mathrm{j}))=\sum \mathrm{wx} * \operatorname{ax}(\mathrm{i}, \mathrm{j}) / \mathrm{bx}(\mathrm{i}, \mathrm{j}) \quad(2) \\
\text { wx: Weight-factor for attributes of type } \mathrm{x} \\
\text { ax: Number of common, type } \mathrm{x} \text { attributes from item } \mathrm{i} \text { and } \mathrm{j} \\
\text { bx: Lower number of type } \mathrm{x} \text { attributes from item } \mathrm{i} \text { and } \mathrm{j}
\end{array}
$$

As previously mentioned, attributes can give different amount of information. For example, the fact an item was produced in USA or that it was produced in the year 2004 does not really tell us anything about the item's content. However, if the same director for example produced the item there is a considerable possibility that the items will share some 
similarities. We used linear regression method on 100 randomly selected ratings to find the optimal weight-factor that would produce the minimum error in the rating prediction. The result of the regression method is the following weights:

W1: Genre 30.08

W2: Emotion 41.16

W3: Country 10.54

W4: Cast 9.08

W5:9.14

Example 3: let's assume that we have the following two items that we want to compare: "The Terminator (1984)" and "Terminator II (1991)" that presumably should give a relatively high similarity value since they have many of the same characteristics. See Table 5.

TABLE V. PROPORTION OF Similar ATtRIBUTES - EXAMPLE

\begin{tabular}{|c|l|l|l|}
\hline Title & $\begin{array}{c}\text { The Terminator } \\
\text { (1984) }\end{array}$ & $\begin{array}{c}\text { The Terminator II } \\
\text { (1991) }\end{array}$ & Common \\
\hline Genre & $\begin{array}{l}\text { Action, Sci-Fi, } \\
\text { Thriller }\end{array}$ & $\begin{array}{l}\text { Action, Sci-Fi, } \\
\text { Thriller, Adventure }\end{array}$ & $3 / 4$ \\
\hline colors & Black, red & Black, red & $2 / 2$ \\
\hline Cast & $\begin{array}{l}\text { Arnold } \\
\text { Schwarzenegger, } \\
\text { Michael Biehn, } \\
\text { Linda Hamilton }\end{array}$ & $\begin{array}{l}\text { Arnold } \\
\text { Schwarzenegger, } \\
\text { Michael Biehn, Linda } \\
\text { Hamilton }\end{array}$ & $3 / 15$ \\
\hline Year & 1984 & 1991 & $0 / 1$ \\
\hline Country & USA & USA & $1 / 1$ \\
\hline
\end{tabular}

After applying equation (2) on example 3, the similarity between the two movies is $82.48 \%$ as calculated below:

$$
\begin{aligned}
& \operatorname{Sim}=0.3008 * \frac{3}{4}+0.4116 * \frac{2}{2}+0.908 * \frac{3}{15}+0.1054 * \frac{1}{1}+0.0914 * \frac{0}{1} \\
& \operatorname{Sim}=92.42 \%
\end{aligned}
$$

After calculated the similarity value for each item pair updated the value and the total number of common attributes in the item-item-matrix, $\mathrm{N}$ nearest neighbors with a minimum threshold of 5 common attributes that the neighbor has to have in common with the neighbor in order for it to qualify and be used for the prediction. The threshold limit was found by a few simple experiments and then evaluating the mean absolute error between the prediction and the real rating. The size of the threshold controls a balance between the quality of the neighbors and the amount of neighbors we use for the prediction. If a threshold is chosen too low (e.g. common > 2) the prediction may use item-neighbors that have high similarity value only because of bad/short descriptions and are irrelevant in relation to other items that have more attributes in common but still manage to get a lower similarity value. However if the threshold is chosen too high (e.g. common > 40) the prediction will possibly be made using too few neighbors or perhaps even no neighbors qualify which means that we are unable to make the prediction, at least from the item-CF side.

Example 4: Let's assume that seven movies according to item profiles by using equation (2), we found similarity between items, and using matrix for easy presentation similarity between movies, see Table 6 .

\begin{tabular}{|c|c|c|c|c|c|c|c|}
\hline & $\vec{\Xi}$ & $\stackrel{N}{\Xi}$ & $\stackrel{m}{\Xi}$ & $\stackrel{+}{\Xi}$ & $\begin{array}{l}10 \\
\mathbb{E} \\
\stackrel{\Xi}{\Xi}\end{array}$ & 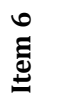 & $\stackrel{\mathbb{E}}{\underline{\Xi}}$ \\
\hline Item 1 & 1 & 0.48 & 0.84 & 0.74 & 0.23 & 0.69 & 0.95 \\
\hline Item 2 & & 1 & 0.98 & 0.19 & 0.56 & 0.61 & 0.87 \\
\hline Item 3 & & & 1 & 0.90 & 0.54 & 0.55 & 0.71 \\
\hline Item 4 & & & & 1 & 0.72 & 0.43 & 0.70 \\
\hline Item 5 & & & & & 1 & 0.51 & 0.63 \\
\hline Item 6 & & & & & & 1 & 0.81 \\
\hline Item 7 & & & & & & & 1 \\
\hline
\end{tabular}

TABLE VI. ITEM-ITEM SIMILARITY MATRIX

Above example shows how the Item-Item-Matrix is constructed with the similarity values between the relative items. Example above each similarity value in the subscript style represents the number of common attributes used for each calculation. Fig 8 shows the php code for finding similarity between movies.

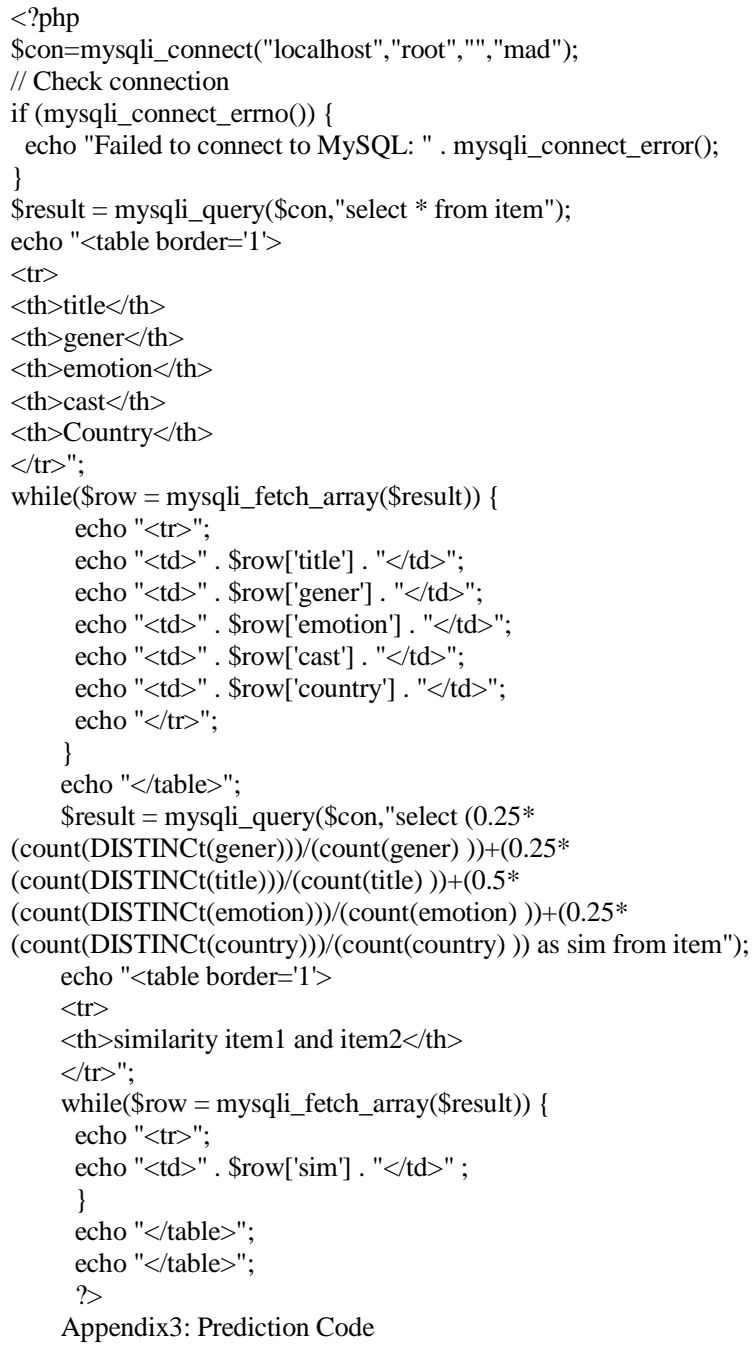


$<$ ?php

\$searchTerm $=$ trim $\left(\$ \_\right.$GET['keyname'] $)$;

if $(\$$ searchTerm $==" ")$

\{

echo "Enter name you are searching for."; exit();

\}

\$host = "localhost";

$\$ \mathrm{db}=$ "mad";

\$user = "root";

\$pwd = "";

\$link = mysqli_connect $($ \$host, \$user, \$pwd, \$db);

\$query = "SELECT 'user id’, 'item id’, ‘rating', ‘emotion` FROM

'rating' WHERE name LIKE ' $\% \$$ searchTerm $\%$ ' and rating $>=3$ and emotion ="action"";

\$results = mysqli_query $(\$$ link, $\$$ query $)$;

if(mysqli_num_rows( (results) $>=1$ )

\{

\$output = "';

while $($ \$row $=$ mysqli_fetch_array $($ \$results $))$

\$output $=$ " $<$ video width='320' height='240' controls $><$ source src='rating/". \$row['vid'] .".mp4' type='video/mp4'></video $>"$. "<br >";

echo \$output;

\}else

echo "There was no matching record for the name " . \$searchTerm;

Fig. 8. php code for item-item similarity

\section{Calculation Prediction (User-Item Matrix)}

For finding prediction, items for each user should be calculated prediction for items per users by using equation (3), and then we can get user-item matrix as presents in Table 7.

$$
\mathrm{P} \mathrm{i}, \mathrm{u}=\frac{\sum \mathrm{Si}, \mathrm{n} * \mathrm{R} \mathrm{u}, \mathrm{n}}{\sum_{\text {si,n: }} \mathrm{Similarity} \mathrm{value}}
$$

si,n: Similarity value between target item $i$ and neighbor $n$

$\mathrm{Ru}, \mathrm{n}$ : Rating from neighbor $\mathrm{n}$

Example 5: for predicting rating user 1 to item 1 we use equation 3, through the neighbor's similarity user's ratings.

Sim user1 with neighbours: 0.52, 0.36, 0.44, 0.5

Rating user1 for items: 5,0,0,1

$$
\mathrm{P} 1=\frac{0.52 * 5+0.36 * 0+0.44 * 4+0.5 * 1}{0.52+0.36+0.44+0.5}=2.67
$$

Sim item1 with neighbours: $0.48,0.84,0.74,0.23,0.69$, 0.95 .

Rating: 3, 0, 2.5, 3.5, 5,0

$$
\mathrm{P} 2=\frac{0.48 * 3+0.84 * 0+0.74 * 2+0.23 * 5+0.69 * 5+0.95 * 0}{0.48+0.84+0.74+0.23+0.69+0.95}=1.91
$$

The final prediction $(\mathrm{P})$ is prediction between $\mathrm{P} 1$ and $\mathrm{P} 2$ as calculated the following:

\begin{tabular}{|c|c|c|c|c|c|c|c|}
\hline & $\stackrel{\vec{\Xi}}{\stackrel{\Xi}{\Xi}}$ & $\stackrel{N}{\Xi}$ & $\stackrel{m}{\Xi}$ & $\stackrel{+}{\Xi}$ & 足 & 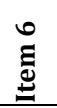 & 氖 \\
\hline User 1 & 2.21 & 2.7 & 2.55 & 3.5 & 3.05 & 3.8 & 3.2 \\
\hline User 2 & 2.8 & 3.9 & 3.5 & 4.5 & 2.75 & 2 & 4 \\
\hline User 3 & 4 & 4.3 & 2.3 & 3.5 & 2.2 & 3.5 & 2.35 \\
\hline User 4 & 3.2 & 4.1 & 2.71 & 1 & 3.1 & 3.5 & 2.5 \\
\hline User 5 & 1.3 & 4.5 & 5 & 1.2 & 4.1 & 2.6 & 3.3 \\
\hline
\end{tabular}

$$
\mathrm{P}=\frac{P 1 * N 1+P 2 * N 2}{N 1+N 2}=\frac{2.67 * 4+1.91 * 6}{4+6}=2.21
$$

TABLE VII. USER - ITEM PREDICTION

D. Calculate Our Approach New User-Item Matrixes and List Of Prediction

After rating movies from users (see Table 1), and finding prediction for movie's rating from users (see Table 7), we get two user-item matrixes, our approach is combining these two matrixes after apply a condition. The condition is replace ratings table 1 to table 7 with remain 1 and 5, because number 1 meaning the user absolutely dislike the movie and number 5 meaning the user absolutely like the movie, so no need to find predictions, but ratings between 1 and 5 may changed after

\begin{tabular}{|c|c|c|c|c|c|c|c|}
\hline & $\stackrel{-1}{\stackrel{\Xi}{\Xi}}$ & $\stackrel{N}{\Xi}$ & $\stackrel{m}{\Xi}$ & $\stackrel{+}{\Xi}$ & 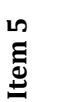 & 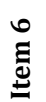 & $\stackrel{N}{\Xi}$ \\
\hline User 1 & 2.21 & 2.7 & 2.55 & 3.5 & 3.05 & 5 & 3.2 \\
\hline User 2 & 5 & 3.9 & 3.5 & 4.5 & 2.75 & 2 & 4 \\
\hline User 3 & 4 & 4.3 & 2.3 & 3.5 & 1 & 3.5 & 2.35 \\
\hline User 4 & 3.2 & 4.1 & 2.71 & 1 & 3.1 & 3.5 & 2.5 \\
\hline User 5 & 1 & 4.5 & 5 & 1.2 & 4.1 & 2.6 & 3.3 \\
\hline
\end{tabular}
more rating from other users that profiles near the target user, as shown in Table 8.

TABLE VIII. USER-ITEM OUR ALGORITHM

In the final step the system list the top movies for target user, the system predict best movies for the target user that like watch it.

\section{E. Review System}

To review the system, these figures below show the user interface in Fig.9, and system framework in Fig.10.

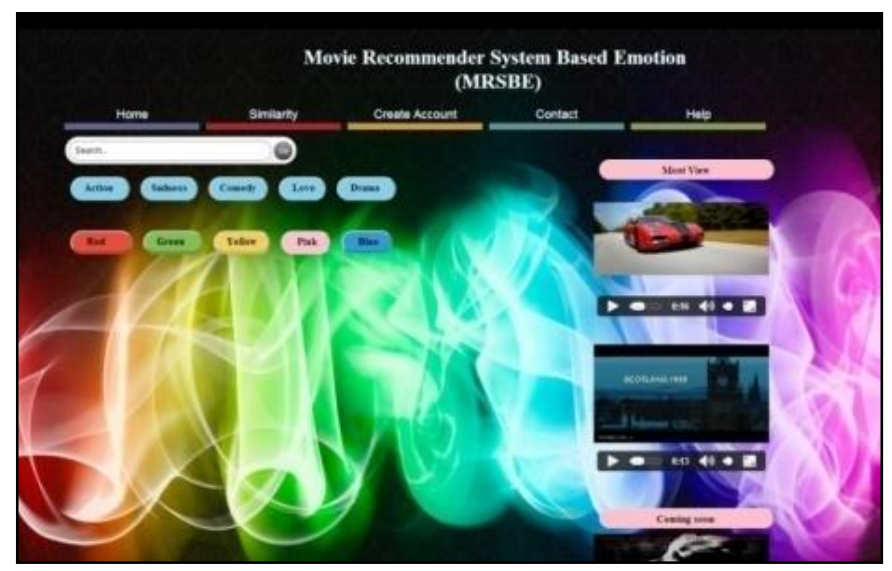

Fig. 9. Interface New Movie Recommender System 


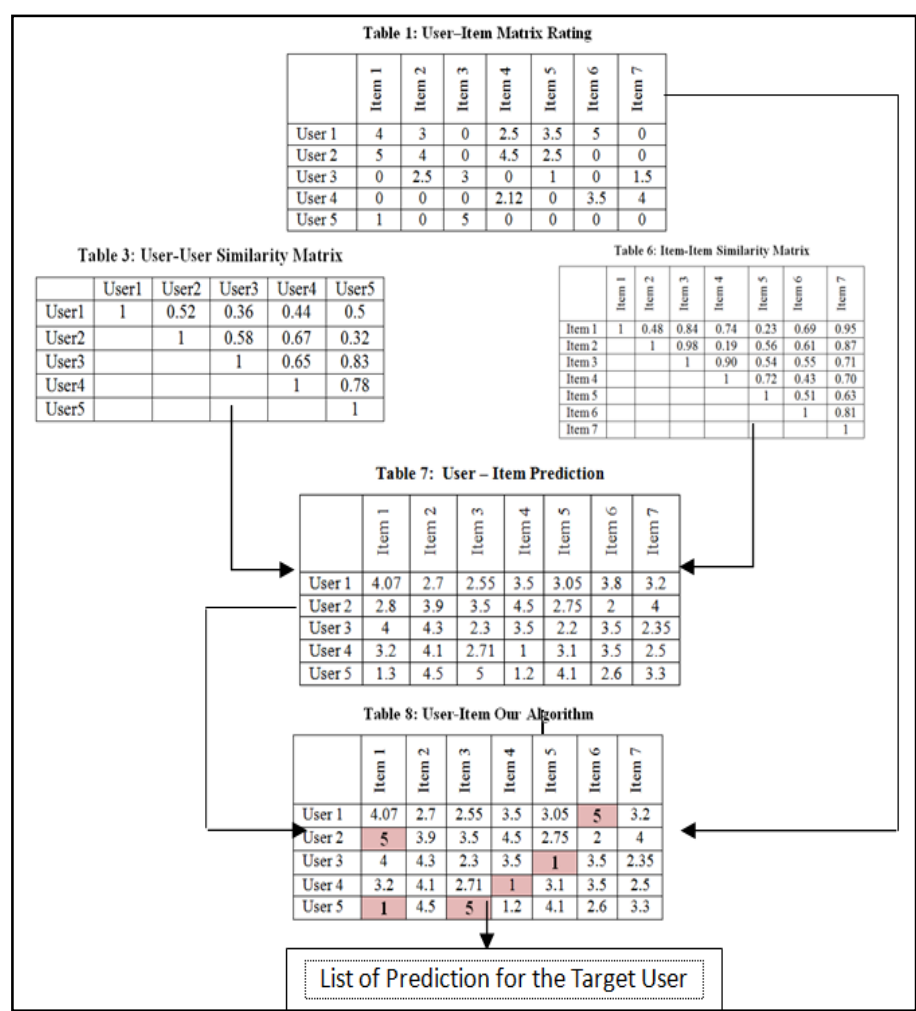

Fig. 10. System Framework

\section{CONCLUSION AND FUTURE WORK}

In this paper we designed a new web recommender system for movies based on emotion. The movies are a complex object and emotions are a human interaction, which is difficult combining together. In this paper, we applied matrix for integrating movie recommendation by hybrid approach, which consists of $\mathrm{CBF}$ and $\mathrm{CF}$ system with emotions detection algorithm and our algorithm. Furthermore our algorithm calculated the user rating 1 and 5 because the users absolutely liked or disliked the movies this system much better recommendations to users because it enables the users to understand the relation between their emotional states and the recommended. We recommend the researchers to improve this idea through: 1) Extracting the movies to finding most using colors by system. 2) Using more than two recommendation techniques to getting best capture of the movies. 3) Using more than three colors to finding human emotions. 4) Design a new algorithm to solving the movie recommender system.

\section{REFERENCES}

[1] Ricci, F., Rokach, L., \&Shapira, B. (2011). "Introduction to recommender systems handbook": Springer.

[2] Eyjolfsdottir, E. A., Tilak, G., \&Li, N.(2010) "MovieGEN: A Movie Recommendation System", UC Santa Barbara: Technical Report.

[3] Shani, G. \&Gunawardana, A. (2011). "Evaluating recommendation systems", in Recommender systems handbook, ed: Springer, 2011, pp. 257-297.

[4] Pazzani, M. J. \&Billsus, D. (2007). "Content-based recommendation systems", in The adaptive web, ed: Springer, 2007, pp. 325-341.

[5] Ho, A. T., Menezes, I. L., \&Tagmouti, Y. (2006) "E-MRS: Emotionbased movie recommender system", in Proceedings of IADIS e-
Commerce Conference. USA: University of Washington Both-ell, pp. 18, 2006.

[6] Joly, A., Maret, P., \&Daigremont, J. (2010) "Enterprise Contextual Notifier, Contextual Tag Clouds towards more Relevant Awareness", in Proceedings of the ACM Conference on Computer Supported Cooperative Work, pp. 531-532, 2010.

[7] Costa, H.(2012) "A Multiagent System Approach for Emotion-based Recommender Systems", PhD proposal, University of Coimbra, Coimbra, Portugal.

[8] Good, N., Schafer, J. B., Konstan, J. A., Borchers, A., Sarwar, B., Herlocker, J., \&Riedl, J. (1999) "Combining collaborative filtering with personal agents for better recommendations", in AAAI/IAAI, pp. 439446, 1999.

[9] Perdew, J. P., Burke, K., \&Ernzerhof, M.(1996) "Generalized gradient approximation made simple", Physical review letters, vol. 77, p. 3865.

[10] Costa, H. \&Macedo, L. (2013). "Emotion-Based Recommender System for Overcoming the Problem of Information Overload", in Highlights on Practical Applications of Agents and Multi-Agent Systems, ed: Springer, 2013, pp. 178-189.

[11] Quijano-Sanchez, L., Recio-Garcia, J. A., \&Diaz-Agudo, B. (2011) "Happymovie: A facebook application for recommending movies to groups", in Tools with Artificial Intelligence (ICTAI), 2011 23rd IEEE International Conference on, pp. 239-244, 2011.

[12] Yang, C.-Y. \&Yuan, S.-T.(2010) "Color Imagery for Destination Recommendation in Regional Tourism".

[13] Rajenderan, A.(2014) "An Affective Movie Recommendation System".

[14] Melville, P., Mooney, R. J., \&Nagarajan, R. (2002) "Content-boosted collaborative filtering for improved recommendations", in AAAI/IAAI, pp. 187-192, 2002.

[15] Nie, D., Hong, L., \&Zhu, T. (2013) "Movie Recommendation Using Unrated Data", in Machine Learning and Applications (ICMLA), 2013 12th International Conference on, pp. 344-347, 2013.

[16] Peleja, F., Dias, P., Martins, F., \&Magalhães, J.(2013) "A recommender system for the TV on the web: integrating unrated reviews and movie ratings", Multimedia systems, vol. 19, pp. 543-558.

[17] Kim, M. \&Park, S. O.(2013) "Group affinity based social trust model for an intelligent movie recommender system", Multimedia tools and applications, vol. 64, pp. 505-516.

[18] Liang, T., Wu, S., \&Cao, D. (2012). "Improved Collaborative Filtering Method Applied in Movie Recommender System", in Emerging Computation and Information teChnologies for Education, ed: Springer, 2012, pp. 427-432.

[19] Jung, K.-Y., Park, D.-H., \&Lee, J.-H. (2004). "Personalized movie recommender system through hybrid 2-way filtering with extracted information", in Flexible Query Answering Systems, ed: Springer, 2004, pp. 473-486.

[20] Fernández-Tobías, I., Cantador, I., \&Plaza, L. (2013). "An Emotion Dimensional Model Based on Social Tags: Crossing Folksonomies and Enhancing Recommendations", in E-Commerce and Web Technologies, ed: Springer, 2013, pp. 88-100.

[21] Lichtenberg, A. J. \&Lieberman, M. A.(1983) "Regular and stochastic motion", Research supported by the US Department of Energy, US Navy, and NSF. New York, Springer-Verlag (Applied Mathematical Sciences. Volume 38), 1983, 516 p., vol. 38.

[22] Gaczynska, M., Rock, K. L., \&Goldberg, A. L.(1993) " $\gamma$-Interferon and expression of MHC genes regulate peptide hydrolysis by proteasomes".

[23] Chinwalla, A. T., Cook, L. L., Delehaunty, K. D., Fewell, G. A., Fulton, L. A., Fulton, R. S., Graves, T. A., Hillier, L. W., Mardis, E. R., \&McPherson, J. D.(2002) "Initial sequencing and comparative analysis of the mouse genome", Nature, vol. 420, pp. 520-562.

[24] Thamm, R.(1992) "Social structure and emotion", Sociological Perspectives, vol. 35, pp. 649-671.

[25] Artymiuk, P., Blake, C., Grace, D., Oatley, S., Phillips, D., \&Sternberg, M.(1979) "Crystallographic studies of the dynamic properties of lysozyme". 